\title{
Predicción de peso de carcasa a la edad de beneficio en cuyes del genotipo Cieneguilla con base a una síntesis de medidas corporales
}

\author{
Prediction of Carcass Weight at the age of Slaughtering in guinea Pigs Of the \\ Cieneguilla GENOTYPE BASED ON A SYNTHESIS OF BODY MEASUREMENTS
}

Pablo Rubio Arias ${ }^{1,2,5}$, Juan Chávez C. ${ }^{3}$, Grimaldo Febres ${ }^{4}$, Hugo Deza C. ${ }^{1}$

\section{Resumen}

El objetivo del presente estudio fue predecir el peso de carcasa (PC) en cuyes (Cavia porcellus) a la edad de beneficio ( $16 \pm 2$ semanas), tomando como referencia sus medidas biométricas. Se tomaron las siguientes medidas y pesos antes y después del beneficio a 150 cuyes machos del genotipo Cieneguilla: peso vivo al beneficio (PV), largo de cuerpo (LC), largo de cabeza (LCA), ancho de cabeza (AC), largo de lomo (LL), ancho de lomo $(\mathrm{AL})$, perímetro torácico $(\mathrm{PT})$, perímetro de muslo $(\mathrm{PM})$, largo de muslo $(\mathrm{LM})$, perímetro de brazuelo (PB), largo de brazuelo (LB) y cuadrado medio de la grupa (CMG), así como el peso de carcasa (PC). Los datos fueron analizados para determinar la mejor ecuación de regresión y establecer el mejor modelo lineal predictivo del peso de carcasa. Se utilizó el proceso «Step-Wise Regression» del paquete estadístico SAS. Las combinaciones de variables independientes en el modelo revelaron que las variables PV, PT, AC y LL dan una mejor explicación del PC a la edad de beneficio $\left(\mathrm{R}^{2}=0.71 ; \mathrm{Cp}-\right.$ Mallows $\left.=1.63\right)$.

Palabras clave: cuy; rendimiento de carcasa; medidas corporales; selección

\section{Abstract}

The objective of the present study was to predict carcass weight (PC) in guinea pigs (Cavia porcellus) at the age of slaughtering (16 \pm 2 weeks), considering their biometric measurements. The following measures and weights were taken before and after the slaughter of 150 male guinea pigs of the Cieneguilla genotype: live body weight (PV),

\footnotetext{
${ }^{1}$ Programa Doctoral en Ciencia Animal, Universidad Nacional Agraria La Molina, Lima, Perú

${ }^{2}$ Unidad Académica de Agricultura, Silvicultura, Pesca y Veterinaria, Universidad Católica de Cuenca, Ecuador

${ }^{3}$ Departamento Académico de Producción Animal, Facultad de Zootecnia, Universidad Nacional Agraria La Molina, Lima, Perú

${ }^{4}$ Departamento Académico de Estadística e Informática, Universidad Nacional Agraria La Molina, Lima, Perú

${ }^{5}$ Email:dr_rubio75@hotmail.com
}

Recibido: 6 de julio de 2017

Aceptado para publicación: 8 de febrero de 2018 
body length (LC), head length (LCA), head width (AC), loin length (LL), loin width (AL), chest girth (PT), thigh perimeter (PM), thigh length (LM), arm perimeter (PB), arm length (LB), and rump middle square (CMG), as well as the carcass weight (PC). The data were analyzed to determine the best regression equation and to establish the best predictive linear model of carcass weight. The «Step-Wise Regression» process of the SAS statistical package was used. The combinations of independent variables in the model revealed that the variables PV, PT, AC, and LL give a better explanation of the $\mathrm{CP}$ at the benefit age $\left(\mathrm{R}^{2}=0.71\right.$; Cp-Mallows $\left.=1.63\right)$.

Key words: guinea pig; carcass yield; body measurements; selection

\section{INTRODUCCIÓN}

En el Perú y en la mayor parte de los países andinos, el cuy (Cavia porcellus) se utiliza desde épocas ancestrales como fuente de carne, especialmente por los pobladores de los ámbitos rurales del país, sin que haya existido interés en su mejora productiva; sin embargo, a partir de la década del 60 el Estado Peruano promovió la mejora genética de la población nacional de cuyes, para lo cual estableció un programa nacional. Derivado de esto, en la actualidad se cuenta con más de tres razas de cuyes creadas principalmente para mejorar la eficiencia productiva y reproductiva del cuy, así como ensayos de producción de híbridos comerciales para la producción de carne (Chauca, 2007).

En el crecimiento y desarrollo del cuy intervienen diversos procesos biológicos que conducen a cambios anatómicos y adaptaciones fisiológicas y, como consecuencia, el sistema músculo esquelético sufre un notable desarrollo por hipertrofia (Glass, 2005), pudiendo alcanzar los 20 a $25 \mathrm{~cm}$ de longitud corporal, pesos entre 700 a $1200 \mathrm{~g}$ (Vanderlip, 2003) y rendimientos de carcasa entre 67 y $74 \%$ a las 12 semanas edad (Cruz, 2013; Meza et al. 2014).

La comercialización de cuyes se realiza como carcasa completa, con cabeza y patas incluidas, lo que permite suponer que su selección por peso vivo tendría un resultado promisorio en términos de rendimiento de carcasa. Es muy probable que en el futuro mediato el mercado exija una mejor calidad de carcasa, por ejemplo, mayor volumen de carne en el cuerpo; y ante esto, la biometría de regiones específicas, como la pierna, brazuelo y lomo, podría facilitar los procesos de selección de animales con una mayor capacidad productiva de carne y una mejor relación carne/hueso. Considerando que el tercio posterior de los animales concentra la mayor parte del peso corporal $(48 \%$ en bivonos [Martínez et al. 2014] y 28\% en cerdos [De Souza et al., 2015]), comparando con un $18 \%$ en cuyes (Chauca et al., 2005), existiría una significativa brecha que podría ser mejorada mediante una adecuada identificación y selección de animales sobresalientes para este atributo.

Adicionalmente a la longitud del cuerpo, se pueden tomar medidas corporales lineales de diversos segmentos anatómicos y ser conjugadas en índices, para aplicarlos a través de ecuaciones matemáticas, que pretendan describir la conformación del cuerpo $\mathrm{y}$, de esa forma, predecir el peso vivo, y en especial el peso y calidad de carcasa a la edad del beneficio. Así mismo, se puedeevaluar el desempeño del fenotipo, examinar las relaciones entre caractaerísticas económicas, el rendimiento reproductivo y lasinteracciones entre la herencia y el medio ambiente (Agamy et al., 2015; Anye et al., 2017). 
En los programas de mejoramiento genético de diversas especies de animales domésticos se aplican herramientas predictivas cuantitativas que facilitan realizar una adecuada selección de los animales en función de sus valores de cría con el fin de incrementar genéticamente su eficiencia productiva y reproductiva. En razón a ello, el presente trabajo tuvo como objetivo predecir el peso de la carcasa de cuyes a la edad de beneficio (16 \pm 2 semanas) con base a la biometría corporal y al peso vivo al momento del beneficio. Además, se determinó el rendimiento de carcasa con base a la relación entre el peso vivo antes del beneficio y el peso de la carcasa.

\section{Materiales y Métodos}

El estudio se desarrolló entre mayo y julio de 2016 en el Centro de Beneficio de la Facultad de Zootecnia de la Universidad Nacional Agraria la Molina (Lima, Perú), con 150 cuyes machos del genotipo «Cieneguilla» de una edad de $16 \pm 2$ semanas, que fueron criados en la Granja Experimental Cieneguilla, perteneciente a la misma universidad.

Mediante el uso de una balanza analítica de precisión $( \pm 1 \mathrm{~g})$, una regla Vernier Caliper milimétrica $( \pm 0.02 \mathrm{~mm})$ y una cinta métrica adaptada, se realizaron diversas mediciones antes y después del beneficio. El peso vivo (PV) fue registrado tras un ayuno de 12 horas; inmediatamente después del beneficio se registró el peso de carcasa (PC) y las siguientes medidas biométricas: largo de cuerpo (LC), tomado desde la punta de la nariz hasta la última vértebra coccígea; perímetro torácico $(\mathrm{PT})$, tomado por detrás de los brazos a la altura de la escápula abrazando la caja torácica; largo de lomo (LL), tomado desde el borde caudal de la última costilla hasta la última vértebra coccígea; ancho de lomo (AL), tomado a la altura de las vértebras lumbosacras, entre las apófisis transversa derecha e izquierda; ancho de cabeza (AC), tomando la distancia entre los arcos zigomáticos derecho e izquierdo; largo de cabeza (LCA), tomado desde la punta de la nariz hasta la articulación atlanto-occipital; perímetro de muslo (PM), tomado por encima de la articulación fémoro-tibial-rotuliana, rodeando todo el muslo; largo de muslo (LM), tomado desde la articulación fémoro-tibial hasta la articulación fémoro-rotuliana; perímetro de brazuelo (PB) por encima de la articulación del codo; largo de brazuelo (LB), tomado desde la articulación húmero-radialcubital hasta la articulación escápulo-humeral; cuadrado medio de la grupa (CMG), tomado desde la patela izquierda hacia la derecha. La carcasa incluyó la cabeza, miembros anteriores y posteriores, patas y vísceras rojas (corazón, pulmón, hígado y riñones).

Para determinar la mejor ecuación de predicción se utilizó el procedimiento «Stepwise Regression» del programa estadístico SAS (Beal, 2005), que permite identificar la ecuación de regresión con mejor bondad de ajuste para estimar el peso de carcasa, justificado en la implementación tradicional del método de selección de las variables independientes por etapas forward $y$ backward, y la conjugación de ambas Stepwise, basados en el grado de contribución de las variables referidas, que pueden ser añadidas o eliminadas de acuerdo a su nivel de significancia $(\alpha=0.01)$.

Se obtuvieron los coeficientes de correlación para la variable respuesta (PC) y se corroboró el modelo mediante el análisis de los componentes principales, validándose el modelo predictor, quedando el modelo de regresión definido de la siguiente manera:

$\mathrm{Y}_{\mathrm{ij}}=\beta_{0}+\beta_{1} \mathrm{X}_{1}+\beta_{2} \mathrm{X}_{2}+\beta_{3} \mathrm{X}_{3}+\beta_{4} \mathrm{X}_{4}+\ldots \beta_{12} \mathrm{X}_{12}$

donde $\mathrm{Y}_{\mathrm{ij}}$ es la variable respuesta (PC) y $\beta_{1} \mathrm{X}_{1}$ $+\beta_{2} X_{2}+\beta_{3} X_{3}+\beta_{4} X_{4}+\ldots \beta_{12} X_{12}$ son los coeficientes de regresión asociados a cada una de las medidas anatómicas corporales bajo evaluación. 
Cuadro 1. Medidas biométricas de cuyes genotipo "Cieneguilla" antes y después del beneficio (edad de beneficio: $16 \pm 2$ semanas de edad; $n=150$ cuyes)

\begin{tabular}{lcccc}
\hline \multirow{2}{*}{ Variable } & \multicolumn{2}{c}{ Antes } & \multicolumn{2}{c}{ Después } \\
\cline { 2 - 5 } & (media \pm D.E.) & C.V. (\%) & (media \pm D.E.) & C.V. (\%) \\
\hline Peso corporal, g & $1301.8 \pm 187.7$ & 14.4 & $912.0 \pm 139.3$ & 17.3 \\
Largo de cuerpo, cm & $32.9 \pm 1.6$ & 4.9 & $33.5 \pm 1.6$ & 4.7 \\
Perímetro torácico, cm & $22.2 \pm 1.5$ & 10.0 & $19.8 \pm 1.6$ & 8.0 \\
Perímetro de muslo, cm & $10.9 \pm 0.8$ & 7.6 & $11.7 \pm 1.0$ & 8.4 \\
Largo de muslo, cm & $6.7 \pm 0.7$ & 10.6 & $7.0 \pm 0.5$ & 7.6 \\
Perímetro de brazuelo, cm & $8.3 \pm 0.8$ & 9.8 & $8.0 \pm 0.9$ & 10.7 \\
Largo de brazuelo, cm & $4.6 \pm 0.6$ & 12.1 & $4.9 \pm 0.5$ & 10.4 \\
Largo de cabeza, cm & $7.2 \pm 0.6$ & 8.8 & $6.6 \pm 0.6$ & 9.2 \\
Ancho de cabeza, cm & $3.8 \pm 0.4$ & 11.6 & $3.7 \pm 0.4$ & 11.6 \\
Largo de lomo, cm & $13.5 \pm 2.0$ & 14.8 & $12.7 \pm 1.8$ & 14.2 \\
Ancho de lomo, cm & $3.7 \pm 0.6$ & 15.6 & $3.5 \pm 0.4$ & 12.8 \\
Cuadrado medio de la & $14.8 \pm 1.9$ & 12.6 & $14.5 \pm 1.4$ & 9.8 \\
grupa, cm & & & & \\
\hline
\end{tabular}

D.E.: desviación estándar; C.V.: coeficiente de variación

\section{Resultados}

Los resultados de las medidas biométricas tomadas antes y después del beneficio de cuyes del genotipo Cieneguilla beneficiados a las $16 \pm 2$ semanas de edad se muestran en el Cuadro 1. Las correlaciones del peso de la carcasa con las medidas biométricas se muestran en el Cuadro 2. Se puede observar que el peso vivo antes del beneficio tuvo la más alta correlación positiva con el peso de la carcasa $(r=0.76 ; p<0.01)$. Otras medidas biométricas como el perímetro de tórax, largo de cuerpo, largo de lomo, ancho de lomo y cuadrado medio de la grupa tuvieron correlaciones medias con el peso de carcasa, mientras que los valores más bajos de correlación fueron observados entre el largo de muslo, largo de brazo, largo de cabeza y ancho de cabeza con el peso de carcasa.
En base a las medidas biométricas se elaboró un modelo de regresión para estimar el peso de carcasa a través del procedimiento «Step-Wise». Este modelo señaló que el peso vivo, el perímetro de tórax, el ancho de cabeza y el largo de lomo pueden ser usados en la mejor ecuación de predicción del peso de la carcasa $\left(Y_{i}=-158.89+0.47 P V+\right.$ $23.49 P T-32.99 A C+5.09 L L ; \mathrm{R}^{2}=0.71$; Cp-Mallows $=1.63 ; \mathrm{p}<0.01)$ de cuyes del genotipo Cieneguilla. Dada la dificultad de obtener medidas confiables del largo de lomo, se buscó una alternativa excluyendo esta medida, quedando la ecuación $Y_{i}=-137.98$ $+0.46 P V+26.38 P T-36.11 A C\left(\mathrm{R}^{2}=0.70\right.$; Cp-Mallows $=1.79 ; \mathrm{p}<0.01$ ).

Finalmente, se estimó el rendimiento de carcasa con base a la relación existente entre el peso vivo antes del beneficio con el peso de la carcasa después del beneficio, 
Cuadro 2. Coeficientes de correlación entre las medidas biométricas y el peso de carcasa de cuyes del genotipo Cieneguilla al momento del beneficio

\begin{tabular}{lc}
\hline & $\begin{array}{c}\text { Peso de } \\
\text { carcasa }\end{array}$ \\
\hline Peso vivo & $0.76^{* *}$ \\
Largo del cuerpo & $0.43^{* *}$ \\
Perímetro torácico & $0.49^{* *}$ \\
Perímetro de muslo & $0.28^{* *}$ \\
Largo de muslo & -0.1 \\
Perímetro de brazuelo & $0.30^{* *}$ \\
Largo de brazuelo & 0.02 \\
Largo de cabeza & 0.03 \\
Ancho de cabeza & -0.11 \\
Largo de lomo & $0.45^{* *}$ \\
Ancho de lomo & $0.43^{* *}$ \\
Cuadrado medio de la grupa & $0.53^{* *}$ \\
\hline$* *$ p<0.01 &
\end{tabular}

obteniéndose un valor de $70.1 \%$ para los cuyes del genotipo Cieneguilla.

\section{Discusión}

Reportes previos de las medidas biométricas de cuyes al momento del beneficio no han sido reportados. En los resultados obtenidos, los coeficientes de variabilidad se encontraron dentro de los rangos permitidos para estudios de campo. La baja variabilidad observada en las medidas sería el resultado de los constantes procesos de selección (Mavule et al., 2013) que se vienen realizando en estos animales, toda vez que el genotipo Cieneguilla es un genotipo que viene siendo desarrollado por la Universidad Nacional Agraria La Molina con el objetivo de obtener animales de buen peso en el menor tiempo posible. El peso vivo, largo de lomo y cuadrado medio de la grupa tuvieron una relativa mayor variabilidad entre las medidas que se determinaron en el presente estudio, lo cual sería consecuencia de características propias del individuo y de los efectos del medio ambiente (Mavule et al., 2013).

La alta correlación entre el peso vivo con el peso de carcasa sería debido a que una gran proporción del peso vivo lo constituye la carcasa que, en el caso de los cuyes, además de musculatura y estructura ósea, se consideran los apéndices (cabeza y patas) y algunas vísceras (corazón, pulmón, hígado y riñones). Este hecho se ve reflejado en la alta tasa de rendimiento de carcasa observado en el presente estudio. El rendimiento de carcasa observado se encuentra dentro del rango de valores reportado por Chauca et al. (2005) en cuyes de raza Perú a las 8 semanas (72.6\%) y Aliaga et al. (2009), quienes reportan rendimientos que fluctúan entre 67.4 y $75.3 \%$ en cuyes mejorados. Estos autores mencionan que este rendimiento se encuentra influenciado por la genética del animal, la edad, la alimentación y la castración. No obstante, la genética tendría el mayor efecto, tal como lo reportan Abdullah et al. (2010). Sarria (2011) y Arbulú y Del Carpio (2015) indican que el genotipo de los cuyes influye sobre el porcentaje de rendimiento, especialmente si se trata de una raza o línea carnicera, o de un cruzamiento. Por otro lado, Cruz (2013) reporta $67 \%$ de rendimiento en cuyes producto de cruce, valor inferior al obtenido en el presente estudio, y rendimientos aún más bajos en cuyes criollos, donde Aliaga et al. (2009) reportan 54.3\% de rendimiento

La edad de los cuyes también podría haber tenido un efecto sobre el peso de carcasa, pues en cuyes del mismo genotipo al utilizado en el presente estudio se reportó un rendimiento del $73 \%$ en cuyes de $16 \pm 3$ semanas de edad (Camino e Hidalgo, 2014), valor que supera ligeramente al observado en el presente estudio. Esto podría ser un indicativo que el rendimiento óptimo de carcasa también dependería de la edad de beneficio (Huerta Leidenz et al., 2013). 
Conclusiones

- El peso vivo, largo del lomo, perímetro torácico y ancho de cabeza fueron las medidas biométricas que permitieron obtener la mejor ecuación predictiva del peso de carcasa a la edad del beneficio en cuyes genotipo Cieneguilla.

- La biometría es una herramienta útil y al alcance de los criadores que puede ser usada para estimar el peso de carcasa de cuyes a la edad del beneficio.

\section{Literatura Citada}

1. Abdullah AY, Kridli RT, Shaker MM, Obeidat MD. 2010. Investigation of growth and carcass characteristics of pure and crossbreed Awassi lambs. Small Ruminant Res 94: 167-175. doi: 10.1016/ j.smallrumres.2010.08.005

2. Agamy R, Abdel-Moneim A, Abd-Alla M, Abdel-Mageed I, Ashmawi G. 2015. Using linear body measurements to predict body weight and carcass characteristics of three Egyptian fattailed sheep breeds. Asian J Anim Vet Adv 10: 335-344. doi: 10.3923/ ajava.2015.335.344

3. Anye NH, Manjeli Y, Ebangi AL. 2017. A method of live weight evaluation for local guinea pigs (Cavia porcellus L.) of the western highlands of Cameroon: synthesis of linear body measurements. Livestock Res Rural Dev 22(10). [Internet]. Available in: http:// www.lrrd.org/lrrd22/10/anye22184.htm

4. Arbulú CA, Del Carpio PA. 2015. Rendimiento y contenido graso de cuyes ( $c a-$ via porcellus) mejorados, sacrificados a la octava y duodécima semana de edad. Rev Invest Cultura 4(1): 20-32.

5. Beal DJ. 2005. $\mathrm{SAS}^{\circledR}$ code to select the best multiple linear regression model for multivariate data using information criteria. Science Applications International Corporation. USA. [Internet].
Available in: http://analytics.ncsu.edu/ sesug/2005/SA01_05.pdf

6. Bennet C. 2009. Using heritability for genetic improvement. USA: VCE Publications. [Internet]. Available in: http://pubs.ext.vt.edu/404/404-084/404084.html

7. Cam MA, Olfaz, M, Soydan E. 2010. Body measurements reflect body weights and carcass yields in Karayaka sheep. Asian J Anim Vet Adv 5: 120-127. doi: 10.3923/ajava.2010.120.127

8. Camino J, Hidalgo V. 2014. Evaluación de dos genotipos de cuyes (Cavia porcellus) alimentados con concentrado y exclusión de forraje verde. Rev Inv Vet Perú 25: 190-197. doi: 10.15381/ rivep.v25i2.8490

9. Chauca L. 2007. Realidad y perspectiva de la crianza de cuyes en los paises andinos. En: Res XX Reunión ALPA. Cusco, Perú.

10. Clow KA, Giraud GD, Ogden BE, Brooks VL. 2002. Pregnancy alters hemodynamic responses to hemorrhage in conscious rabbits. Am J Physiol Heart Circ Physiol 284: H1110-H-1118. doi: 10.1152/ajpheart.00626.2002

11. Chauca FL, Muscari J, Higahonna R. 2005. Informe final sub-proyecto generación de líneas mejoradas de cuyes de alta productividad. INIA-INAGRO. Lima: INIA. $164 \mathrm{p}$.

12. Cruz MM. 2013. Comportamiento productivo de progenies $\mathrm{F}_{2}$ de cuatro cruzamientos entre grupos raciales de cuyes (Cavia porcellus) de hembras $\mathrm{F}_{1}$ con machos Macabeo y peruano mejorado. Tesis de Ingeniero Agrónomo. Quito: Univ. Central del Ecuador. $80 \mathrm{p}$.

13. De Souza D, Alvarado M, Steffens J. 2015. Qualidade da carcça e rendimento de cortes suínos com o uso de ractopamina. Ciênc Rural 45: 1503-1508. doi: 10.1590/0103-8478cr20140625

14. [FAO] Food and Agriculture Organization of the United Nations. 2012. Phenotypic characterization of animal genetic resources. FAO Animal 
Production and Health Guidelines No. 11. Rome. [Internet]. Available in: http:// www.fao.org/docrep/015/i2686e/ i2686e00.pdf

15. Glass DJ. 2005. Skeletal muscle hypertrophy and atrophy signaling pathways. Int J Biochem Cell Biol 37: 19741984. doi: 10.1016/j.biocel.2005.-04.018

16. Huerta Leidenz $N$, Hernández $O$, Rodas González A, Ordóñez, J, Pargas HL, Rincón E, Del Villar A, Bracho B. 2013. Peso corporal y rendimiento en canal según clase sexual, tipo racial, condición muscular, edad y procedencia de bovinos venezolanos. Nacameh 7: 7596.

17. Mavule BS, Muchenje V, Bezuidenhout CC, Kunene NW. 2013. Morphological structure of Zulu sheep based on principal component analysis of body measurements. Small Ruminant Res 111: 23-30. doi: 10.1016/j.smallrumres.2012.09.008

18. Martínez OR, Núñez L, Castro L, Rodríguez MI, Álvarez, R, Florentín-
A, Ramírez L, Pereira W. 2014. Uso de correlación estadística para el estudio morfométrico de bovinos para carne: caso Pampa Chaqueño. Compen Cienc Vet 04(2): 26-32.

19. Meza GA, Cabrera RP, Moran JJ, Meza FF, Cabrera CA, Meza CI, Meza JS, Cabanilla MG, López FX, Pincay JL, Bohórquez T, Ortiz J. 2014. Mejora de engorde de cuyes (cavia porcellus $L$ ) a base de gramineas $\mathrm{y}$ forrajeras arbustivas tropicales en la zona de Quevedo, Ecuador. Idesia 32(3): 7580. doi: $10.4067 / \mathrm{S} 0718-34292014000$ 300010

20. Sarria BJ. 2011. El cuy crianza tecnificada. Manual técnico en cuyicultura N. ${ }^{\circ}$ 1. Lima: UNALM. 64 p.

21. Vanderlip S. 2003. The guinea pig handbook. New York, USA: Barrons. 161 p.

22. William GH. 2010. Understanding and using quantitative genetic variation. Phil Trans R Soc B Biol Sci 365: 73-85. doi: 10.1098/rstb.2009.0203 